\title{
Inkjet-Printed PEI FET-Type Humidity Sensor Having a Horizontal Floating-Gate
}

\author{
Meile Wu ${ }^{1}$, Jongmin Shin ${ }^{1}$, Yoonki Hong ${ }^{1}$, Dongkyu Jang ${ }^{1}$, Xiaoshi Jin ${ }^{2}$, and Jong-Ho Lee ${ }^{1}$ \\ ${ }^{1}$ Department of Electrical and Computer Engineering and Inter-University Semiconductor Research \\ Center, Seoul Nation University, Seoul 151-742, Republic of Korea, \\ 2 College of Information Science and Engineering, Shenyang University of Technology, Shenyang \\ 110870, China, \\ jhl@snu.ac.kr
}

\begin{abstract}
:
In this paper, a field-effect transistor (FET) humidity sensor having horizontal floating-gate (FG) and control-gate (CG) is investigated. Branched polyethylenimine (PEI) is formed on the FET platform for the humidity detection. The inkjet printing process is adopted to deposit the sensing layer, which is convenient and low cost. The humidity sensing properties of the proposed sensor are measured at room temperature. The response, response time and recovery time of the sensor are $415 \%, 3 \mathrm{~min}$ and $13 \mathrm{~min}$ for $20 \%$ relative humidity $(\mathrm{RH})$, respectively. The sensing mechanism is also explained from the perspective of the ion motions in the sensing material.
\end{abstract}

Key words: PEI, inkjet printing, FET gas sensor, humidity detection, room temperature.

\section{Experimental}

The structure of the proposed metal-oxidesemiconductor field-effect transistor (MOSFET) humidity sensor is shown in Fig. 1. Fig. 1 (a) and (b) are the microscopic images of the sensor without and with printed sensing material, respectively. Fig. 1 (c) and (d) show the 2D schematic cross-sectional views cut along line $A-A^{\prime}$ and $B-B^{\prime}$ in (a), respectivley. The formula of $\mathrm{PEI}$ is also shown in (c). The interdigitated $F G$ and $C G$ are formed horizontally to increase the coupling ratio between them. In this paper, pMOSFET sensor platform is adopted. Brenched PEI, which has primary, secondary, and tertiary amines, is deposited on the surface of the platform by inkjet printing process to serve as the sensing material. For preparing a PEI ink, Wt. 50\% branched PEl aqueous solution (number average molecular weight 1200) is diluted by D.I. water to $\mathrm{Wt}$. $1 \%$ to reduce the viscosity of the solution because ink with 1 10 cps viscosity is suitable for inkjet printing [1]. A humid gas sample is obtained by running the dry $\mathrm{N}_{2}$ through a bubbler filled with D.I. water and the reference gas is dry $\mathrm{N}_{2}$. All electrical measurements were carried out with an Agilent B1500A at room temperature.

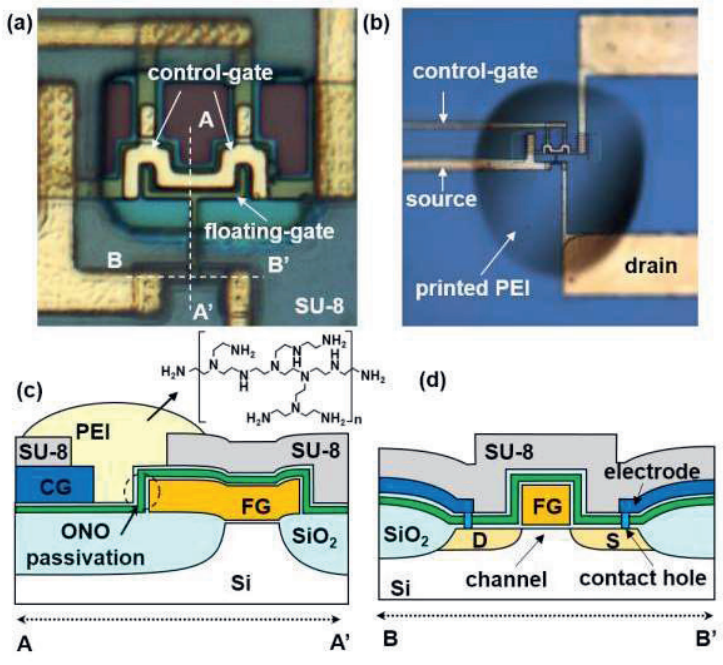

Fig. 1. (a) and (b) show the microscopic images of the sensor without and with printed PEI, respectively. (c) and (d) show the $2 D$ schematic cross-sectional views cut along line $A-A^{\prime}$ and $B-B^{\prime}$ in (a), respectively. The formula of PEI is shown in (c).

\section{Results and Discussion}

Fig. 2 and Fig. 3 show the drain-current versus gate-voltage (ID-VGS) curve on a log scale and gate-current versus gate-voltage ( $\left.I_{\mathrm{G}}-V_{\mathrm{GS}}\right)$ curve on a linear scale of the sensor, respectively. IG$V_{G S}$ curves on a log scale are also plotted in Fig. 2 for a comparison with $I_{D}-V_{G S}$ curves. In Fig. 2, 
the subthreshould swing (SS) and off-state ID increase as $\mathrm{RH}$ increases from zero to $40 \%$. In addition, the gate leakage current $\left(I_{\mathrm{G}}\right)$ also becomes larger with $\mathrm{RH}$ in Fig.3.

Fig. 4 shows the transient humidity sensing performance of the sensor. Dry and $20 \% \mathrm{RH} \mathrm{N} 2$ gases are introduced into the measurement chamber, alternatively. The $\left|I_{D}\right|$ before humidity sensing is initialized to $2 \mathrm{nA}$ with $V_{\mathrm{GS}}=0.1 \mathrm{~V}$ and $V_{D S}=-0.5 \mathrm{~V}$ in dry $\mathrm{N}_{2}$. The $\left|I_{D}\right|$ increases with the increasing humidity. We define the response $(S)$ of a sensor as eq. (1). ID and ID' represent the drain current in dry and humid $\mathrm{N}_{2}$, respectively. The response time ( $t_{\mathrm{RES}}$ ) and recovery time $\left(t_{R E C}\right)$ are defined as the rise time of $\left|I_{D}\right|$ to $90 \%$ of its maximum value and the fall time to $10 \%$ of the difference between the maximum and reference currents. The $S, t_{\mathrm{RES}}$ and $t_{\mathrm{REC}}$ of the proposed sensor are $415 \%, 3 \mathrm{~min}$ and $13 \mathrm{~min}$, respectively.

$S=\frac{\left|I_{D}\right|-\left|I_{D}{ }^{\prime}\right|}{I_{D}} \times 100 \%$ (1)

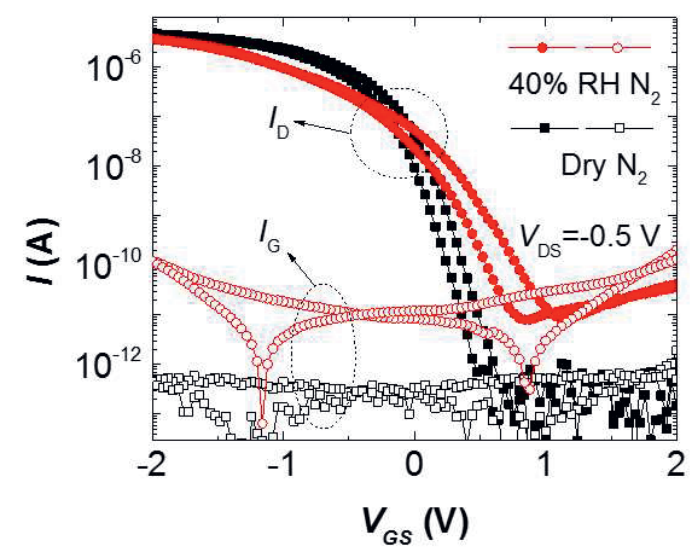

Fig.2. Double sweep $I_{D}-V_{G S}$ and $I_{G}-V_{G S}$ curves on a log scale of the sensor in dry and $40 \% \mathrm{RH} \mathrm{N}_{2}$ gases.

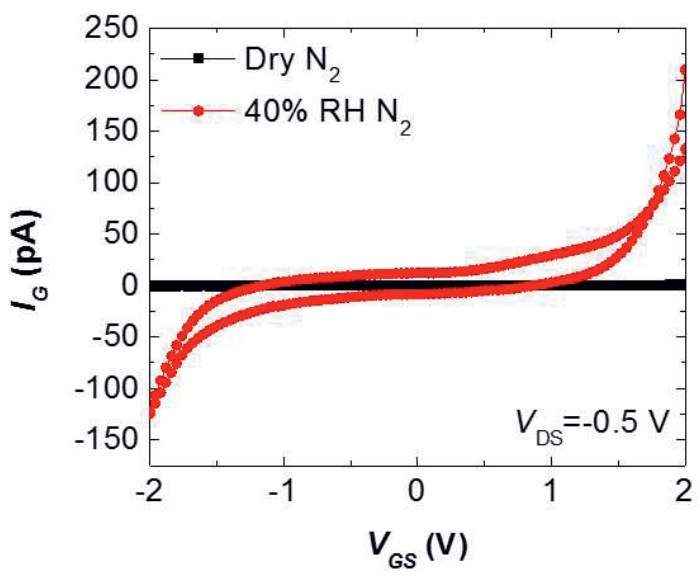

Fig.3. Double sweep $I_{G}-V_{G S}$ curves on a linear scale of the sensor in dry and $40 \% \mathrm{RH} \mathrm{N}_{2}$ gases.

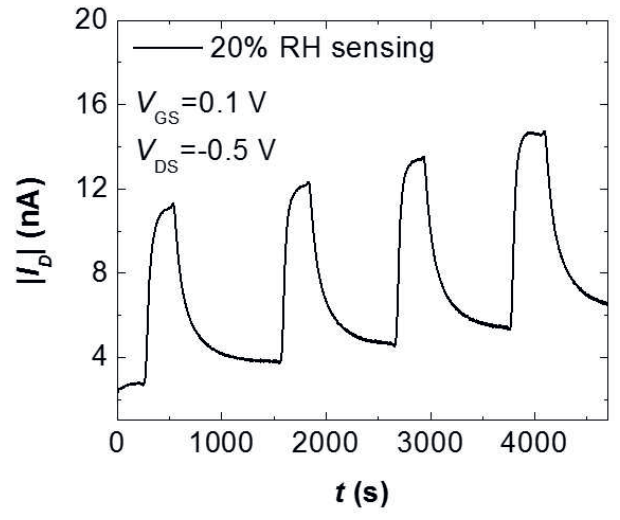

Fig.4. Transient humidity sensing performance of the sensor at room temperature.

During humidity sensing, water molecules adsorbe in PEI due to the hydrogen bond basic character of PEI [2], and partially protonate the aminies of PEI by donating protons [3]. The reactions can be discribed as eq. (2), (3), and (4) for primary, sencondary, and tertiary amines of $\mathrm{PEI}$, respectively. The "R" denotes alkyl groups. As a result, the number of ions in PEI increases and their motions induce the large off state $I_{D}$ and the $S S$. The high $I_{G}$ in humid $N_{2}$ is also attributed to the displacement current caused by moving ions. In Fig. 2, $\left|I_{D}\right|$ increases with humidity in the subthreshold region of $I_{D-}$ $V_{G s}$ curve. Therefore, as $|/ D|$ is initialized to $2 \mathrm{nA}$, it increases with $\mathrm{RH}$ as shown in Fig. 4.

$$
\begin{aligned}
& \mathrm{RNH}_{2}+\mathrm{H}_{2} \mathrm{O} \leftrightarrow \mathrm{RNH}_{3}^{+}+\mathrm{OH}^{-}(2) \\
& \mathrm{R}_{2} \mathrm{NH}+\mathrm{H}_{2} \mathrm{O} \leftrightarrow \mathrm{R}_{2} \mathrm{NH}_{2}^{+}+\mathrm{OH}^{-}(3) \\
& \mathrm{R}_{3} \mathrm{~N}+\mathrm{H}_{2} \mathrm{O} \leftrightarrow \mathrm{R}_{3} \mathrm{NH}^{+}+\mathrm{OH}^{-}(4)
\end{aligned}
$$

\section{Acknowledgements}

This work was supported by the National Research Foundation of Korea (NRF2016R1A2B3009361) and the Brain Korea 21 Plus Project in 2018.

\section{References}

[1] B. Feyssa, C. Liedert, L. Kivimaki, L. S. Johansson, $\mathrm{H}$. Jantunen, and L. Hakalahti, Patterned Immobilization of Antibodies within Roll-to-Toll Hot Embossed Polymeric Microfluidic Channels, PloS one 8, e68918 (2013); doi: 10.1371/journal.pone.0068918

[2] G. E. Collins, L. J. Buckley, Conductive PolymerCoated Fabrics for Chemical Sensing. Synthetic Metals 78, 93-101 (1996); doi: 10.1016/03796779(96)80108-1

[3] X. Gu, F. Meng, G. Liu, H. Zhang, J. Zhou, and S. Ruan, Influences of Surface Capping with Electrostatically Self-Assembled PEI on the Photoresponse of a $\mathrm{TiO}_{2}$ Thin Film, Chemical Communications 49, 6328-6330 (2013); doi: $10.1039 / \mathrm{C} 3 \mathrm{CC} 42745 \mathrm{E}$ 